\title{
Spatiotemporal fragmentation and the uncertainties in the solar rotation law
}

\author{
E. Covas ${ }^{1}$, R. Tavakol ${ }^{\star}, 1$, S. Vorontsov ${ }^{\star \star, 1,2}$, and D. Moss ${ }^{\star \star \star, 3}$ \\ 1 Astronomy Unit, School of Mathematical Sciences, Queen Mary, University of London, Mile End Road, \\ London E1 4NS, UK \\ 2 Institute of Physics of the Earth, B. Gruzinskaya 10, Moscow 123810, Russia \\ 3 Department of Mathematics, The University, Manchester M13 9PL, UK
}

Received 12 March 2001 / Accepted 29 May 2001

\begin{abstract}
Analyses of recent helioseismic data indicate that the dynamical regimes at the base of the convection zone can be different from those observed at the top, having either significantly shorter periods or non-periodic behaviour. Recently spatiotemporal fragmentation/bifurcation has been proposed as a dynamical mechanism to account for the multi-mode behaviour that is possibly observed in the solar convection zone, without requiring separate physical mechanisms with different time scales at different depths. Here we study the robustness of this mechanism with respect to changes to the zero order rotation profile, motivated by the uncertainties of and differences between the various reductions of the helioseimic data. We find that spatiotemporal fragmentation is a common feature of the reductions investigated.
\end{abstract}

Key words. Sun: magnetic fields - torsional oscillations - activity

\section{Introduction}

Recent analyses of the helioseismic data, from both the Michelson Doppler Imager (MDI) instrument on board the SOHO spacecraft (Toomre et al. 2000) and the Global Oscillation Network Group (GONG) project (Antia \& Basu 2000), have provided strong evidence that the previously observed torsional oscillations (e.g. Howard \& LaBonte 1980; Snodgrass et al. 1985; Kosovichev \& Schou 1997; Schou et al. 1998), with periods of about 11 years, penetrate into the convection zone to depths of at least 10 percent in radius.

Further studies of these data have produced interesting, but rather inconsistent results. In particular, Howe et al. (2000b) find evidence for the presence of similar oscillations near the tachocline situated close to the bottom of the convection zone, but with a markedly shorter period of about 1.3 years, whereas Antia \& Basu (2000) do not find such oscillations (whilst allowing the possibility that more irregular variations may be present). Whatever the true dynamical behaviour at these lower levels turns out to be, both these results indicate that the variations in the differential rotation can have different dynamical be-

\footnotetext{
Send offprint requests to: E. Covas,

e-mail: eoc@maths.qmw.ac.uk

* e-mail: reza@maths.qmw.ac.uk

$\star \star$ e-mail: svv@maths.qmw.ac.uk

$\star \star \star ~ e-m a i l: m o s s @ m a \cdot m a n . a c \cdot u k$
}

haviour at the top and the bottom of the solar convection zone, with the oscillations at the bottom having either significantly shorter periods or non-periodic behaviour.

Recently, spatiotemporal fragmentation/bifurcation has been proposed as a dynamical mechanism to account for the possible multi-mode behaviour in different parts of the solar convection zone (Covas et al. 2000a, hereafter CTM1). Evidence for this mechanism was found in the context of a two dimensional axisymmetric mean field dynamo model in a spherical shell, with a semi-open outer boundary condition, in which the only nonlinearity is the action of the azimuthal component of the Lorentz force of the dynamo generated magnetic field on the solar angular velocity. The underlying zero order angular velocity was chosen to be consistent with the recent helioseismic data.

Our initial study (CTM1) suffered from two major shortcomings. The dynamo model employed inevitably contained major approximations, and the zeroth order rotation profile used is bound to include uncertainties, given the uncertainties in the inversion techniques, as well as the short extent in time over which data sets are so far available.

To address the first shortcoming, an extensive study was made of the persistence of the fragmentation following plausible changes in the details of the dynamo model. This study showed that the mechanism remains surprisingly 
robust in presence of a variety of rather severe changes to the model (Covas et al. 2001, hereafter CTM2).

Regarding the second shortcoming, CTM2 also included a preliminary study, allowing the zero order rotational profile to be given by either the inversion of the MDI data reported by Toomre et al. (2000) or of the corresponding GONG data (Howe et al. 2000b). This also showed the mechanism to be robust with respect to such changes to the rotation profile.

Given the significant uncertainties that still remain in the helioseismic measurements, especially given the limited length of the data so far available, it is crucial to study the robustness of the spatiotemporal fragmentation mechanism to a wider range of the rotation profiles consistent with the observations.

\section{Zero order rotation profile}

New measurements of the solar internal rotation with SOHO MDI data have been reported recently by Vorontsov et al. (2001), using novel inversion techniques that are quite different from those employed earlier. Results of a $2 \mathrm{D}$ inversion confirmed the previous findings that the torsional oscillations (migrating zonal flows) penetrate deep into the convection zone. However, given the limited accuracy of the helioseismic data currently available, they did not allow the resolution of these oscillations all the way down to the bottom of the convection zone. The so-called 1.5D inversion procedure has also been used, which consists in measuring separately the consecutive terms $\Omega_{i}(r)$ in the expansion

$\Omega(r, \theta)=\sum_{i=0}^{\infty} \Omega_{2 i+1}(r) \frac{\mathrm{d} P_{2 i+1}(\cos \theta)}{\mathrm{d}(\cos \theta)}$

where $P_{2 i+1}$ is the $(2 i+1)$ th Legendre polynomial, $r$ is the distance from the centre and $\theta$ is the co-latitude.

The 1.5D inversion of Vorontsov et al. (2001) was principally targeted at improvement of the spatial resolution of the rotation profile near and just below the base of the convection zone, in the tachocline region-the region where the dynamo mechanism is most sensitive to the rotation profile, since the largest gradients of the angular velocity appear to be localized there.

An important feature of the solar internal rotation as seen in the helioseismic measurements is that the rapid spatial variation of the angular velocity in the tachocline is governed principally by the first two or three terms in the expansion (1); the contribution of higher terms appears to be an order of magnitude smaller, being essentially undetectable in the helioseismic data available at present. Another important feature is that this dominant part of the variation of the angular velocity in the tachocline with depth and latitude, captured by the first few terms in the expansion (1), does not show any changes with solar activity (Vorontsov et al. 2001). Taken together, these two features suggest considering the torsional oscillations as a small-amplitude, rapidly-varying (in $\theta$ ), time-dependent component of the solar rotation, imposed on a largeamplitude, slowly-varying (in $\theta$ ), nearly-stationary component described by the first terms of the expansion (1).

These considerations show that at least a dominant part of the time-independent (background) component of solar rotation can be evaluated by a simple truncation of the expansion (1) to the first two or three terms, making the truncated expansion a natural candidate for the zero-order rotation profile in the dynamo modelling. This choice might be more consistent than using a time average of the inverted rotation profiles (as in CTM2), since the observational data used in the inversions do not yet cover enough (ca. 5 yr) of a complete solar cycle to allow proper averaging. With the zero-order profile derived from the time-independent part of the data we can also eliminate any possibility that torsional oscillations produced by the dynamo model originate from the time varying zonal flows already present (in time-averaged form) in the background rotation profile.

Thus we here describe a comparative study of the effects of employing zero order rotation profiles, obtained by taking the first two and the first three terms of the expansion (1). These profiles, inferred from the 360d SOHO MDI data set, are shown in Fig. 1.
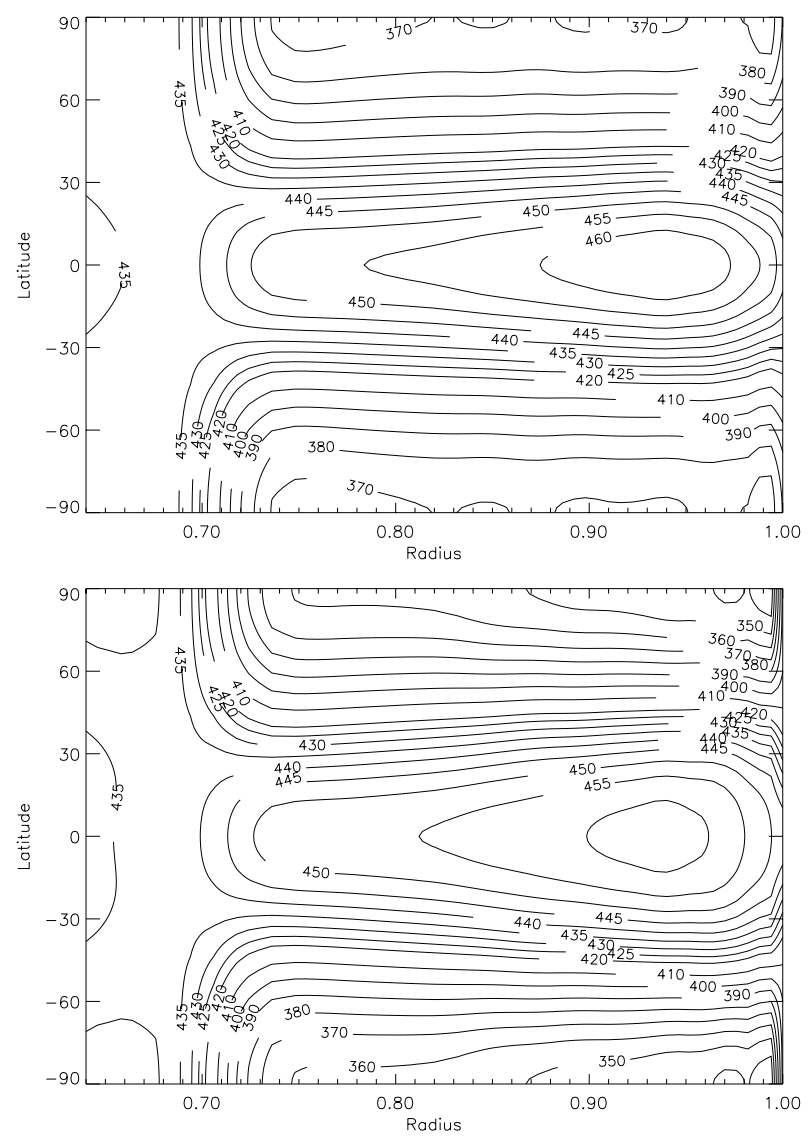

Fig. 1. Isolines of the angular velocity of the solar rotation, obtained from the 1.5-D inversion of the 360d SOHO MDI data set by Vorontsov et al. (2001), given by the first two terms (top panel) and the first three terms (bottom panel) of the expansion (1). Contours are labelled in units of $\mathrm{nHz}$. 


\section{The model}

In order to test the sensitivity of the spatiotemporal fragmentation with respect to the uncertainties in the rotation profile, we used the following dynamo model, which was also used in CTM1,2. We assume that the gross features of the large scale solar magnetic field can be described by a mean field dynamo model, with the standard equation

$$
\frac{\partial \boldsymbol{B}}{\partial t}=\nabla \times(\boldsymbol{u} \times \boldsymbol{B}+\alpha \boldsymbol{B}-\eta \nabla \times \boldsymbol{B}) .
$$

Here $\boldsymbol{u}=v \hat{\phi}-\frac{1}{2} \nabla \eta$, the term proportional to $\nabla \eta$ represents the effects of turbulent diamagnetism, and the velocity field is taken to be of the form $v=v_{0}+v^{\prime}$, where $v_{0}=\Omega_{0} r \sin \theta, \Omega_{0}$ is a prescribed underlying rotation law and the component $v^{\prime}$ satisfies

$$
\frac{\partial v^{\prime}}{\partial t}=\frac{(\nabla \times \boldsymbol{B}) \times \boldsymbol{B}}{\mu_{0} \rho} \hat{\phi}+\nu D^{2} v^{\prime},
$$

where $D^{2}$ is the operator $\frac{\partial^{2}}{\partial r^{2}}+\frac{2}{r} \frac{\partial}{\partial r}+\frac{1}{r^{2} \sin \theta}\left(\frac{\partial}{\partial \theta}\left(\sin \theta \frac{\partial}{\partial \theta}\right)-\right.$ $\left.\frac{1}{\sin \theta}\right)$ and $\mu_{0}$ is the induction constant. The sole nonlinearity in the dynamo equation is the feedback of the azimuthal component of the Lorentz force (Eq. (3)), which modifies only slightly the underlying imposed rotation law, but nevertheless limits the magnetic fields at finite amplitude. The assumption of axisymmetry allows the field $\boldsymbol{B}$ to be split simply into toroidal and poloidal parts, $\boldsymbol{B}=\boldsymbol{B}_{\mathrm{T}}+\boldsymbol{B}_{\mathrm{P}}=B \hat{\phi}+\nabla \times A \hat{\phi}$, and Eq. (2) then yields two scalar equations for $A$ and $B$. Nondimensionalizing in terms of the solar radius $R$ and time $R^{2} / \eta_{0}$, where $\eta_{0}$ is the maximum value of $\eta$, and putting $\Omega=\Omega^{*} \tilde{\Omega}, \alpha=\alpha_{0} \tilde{\alpha}$, $\eta=\eta_{0} \tilde{\eta}, \boldsymbol{B}=B_{0} \tilde{\boldsymbol{B}}$ and $v^{\prime}=\Omega^{*} R \tilde{v}^{\prime}$, results in a system of equations for $A, B$ and $v^{\prime}$. The dynamo parameters are the two magnetic Reynolds numbers $R_{\alpha}=\alpha_{0} R / \eta_{0}$ and $R_{\omega}=\Omega^{*} R^{2} / \eta_{0}$, and the turbulent Prandtl number $P_{\mathrm{r}}=\nu_{0} / \eta_{0} . \Omega^{*}$ is the solar surface equatorial angular velocity and $\tilde{\eta}=\eta / \eta_{0}$. Thus $\nu_{0}$ and $\eta_{0}$ are the turbulent magnetic diffusivity and viscosity respectively, $R_{\omega}$ is fixed when $\eta_{0}$ is determined (see Sect. 4 ), but the value of $R_{\alpha}$ is more uncertain. The density $\rho$ is assumed to be uniform.

Given the ill-determined nature of boundary conditions in astrophysical settings, we make physically motivated choices following CTM1,2. At inner boundary we chose $B=0$, ensuring angular momentum conservation, and an overshoot-type condition on $\boldsymbol{B}_{\mathrm{P}}$ (cf. Moss \& Brooke 2000). At the outer boundary, we used an open boundary condition $\partial B / \partial r=0$ on $B$ and used vacuum boundary conditions for $\boldsymbol{B}_{\mathrm{P}}$ (see Kitchatinov et al. 2000 and CTM1,2 for motivation).

Equations (2) and (3) were solved using the code described in Moss \& Brooke (2000) (where more details are given; see also Covas et al. 2000a) together with the above boundary conditions, over the range $r_{0} \leq r \leq 1$, $0 \leq \theta \leq \pi$. We set $r_{0}=0.64$; with the solar convection zone proper being thought to occupy the region $r \gtrsim 0.7$, the region $r_{0} \leq r \lesssim 0.7$ can be thought of as an overshoot region/tachocline. We note that there is some evidence that the tachocline may be rather narrower than can be directly resolved by the usual inversion techniques. The uncertainties associated with this possibility are difficult to assess, but we do note that the appearance of fragmentation appears to be quite robust with respect to changes in the model (CTM2). In the following simulations we used a mesh resolution of $61 \times 101$ points, uniformly distributed in radius and latitude respectively.

In this investigation, we took the zero order rotation profile $\Omega(r, \theta)$ in the region $0.64 \leq r \leq 1$ to be given successively by the first two and the first three terms of the series (1) given above.

For the alpha-effect, we took $\tilde{\alpha}=\alpha_{\mathrm{r}}(r) f(\theta)$, where $f(\theta)=\sin ^{4} \theta \cos \theta$ (cf. Rüdiger \& Brandenburg 1995) and $\alpha_{\mathrm{r}}=1$ for $0.7 \leq r \leq 0.8$ with cubic interpolation to zero at $r=r_{0}$ and $r=1$, with the convention that $\alpha_{\mathrm{r}}>0$ and $R_{\alpha}<0$. Also, in order to take into account the likely decrease in the turbulent diffusion coefficient $\eta$ in the overshoot region, we allowed a simple linear decrease from $\tilde{\eta}=1$ at $r=0.8$ to $\tilde{\eta}=0.5$ in $r<0.7$. We note, however, that as was shown in CTM2, these latter details of the model are unlikely to remove the possibility of spatiotemporal fragmentations.

\section{Results}

We calibrated our model so that near marginal excitation the cycle period was about 22 years. This determined $R_{\omega}=44000$, corresponding to $\eta_{0} \approx 3.4 \times 10^{11} \mathrm{~cm}^{2} \mathrm{~s}^{-1}$, given the known values of $\Omega^{*}$ and $R$. With the rotation law given by the first three terms of (1), the first solutions to be excited in the linear theory are limit cycles with odd (dipolar) parity with respect to the equator, with marginal dynamo number $R_{\alpha} \approx-3.76$. The even parity (quadrupolar) solutions are also excited at a similar marginal dynamo number of $R_{\alpha} \approx-3.95$. These values did not change significantly when the first two terms of expansion (1) were used. It is arguable that the turbulent Prandtl number is of the order of unity, and we set $P_{\mathrm{r}}=1$. For the parameter range that we investigated, the even parity solutions can be nonlinearly stable. Given that the Sun is observed to be close to an odd (dipolar) parity state, and that previous experience shows that small changes in the physical model can cause a change between odd and even parities in the stable nonlinear solution, we chose to impose dipolar parity on our solutions.

With these parameter values, each of the rotation profiles considered here produced butterfly diagrams which are in qualitative agreement with the observations. The model also successfully produced torsional oscillations that penetrate into the convection zone, in all cases considered, similar to those deduced from recent helioseismic data (Howe et al. 2000a; Vorontsov et al. 2001), and studied in Covas et al. (2000b)

Both of the rotation profiles considered here produced spatiotemporal fragmentation. As examples, the top and the bottom panels of Fig. 2 show the radial contours of the angular velocity residuals $\delta \Omega$ as a function of time for a cut at latitude $30^{\circ}$, with the zero order rotation profile 

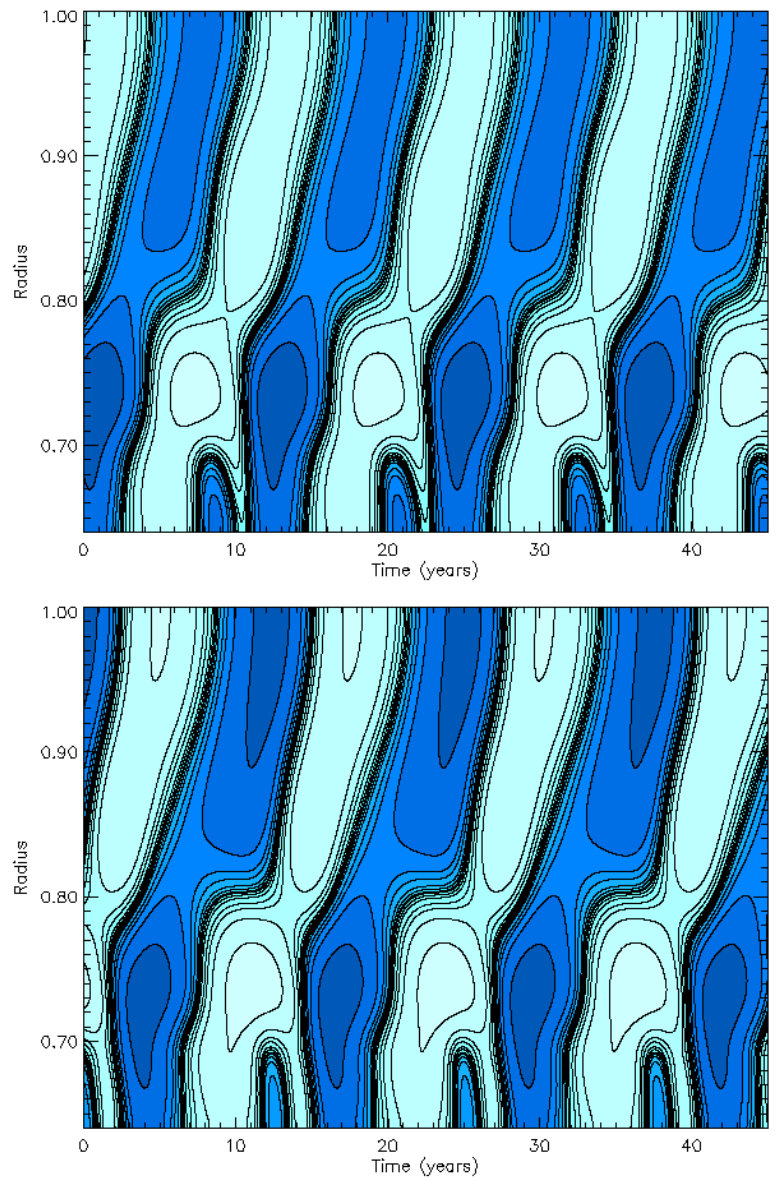

Fig. 2. Radial contours of the angular velocity residuals $\delta \Omega$ as a function of time for a cut at latitude $30^{\circ}$, with the zero order rotation profile obtained by taking the first two (top panel) and the first three terms (bottom panel) in (1). Parameter values are $R_{\alpha}=-20.0, P_{\mathrm{r}}=1.0$ and $R_{\omega}=44000$.

given by the first two and the first three terms in the expression (1), respectively.

To test further this robustness, we also used functions $\Omega_{2 i+1}(r), i=0,1,2$, obtained from an inversion with slightly different regularisation parameters and in a somewhat different frequency range of the input data (Vorontsov et al. 2000). We found no qualitative changes in the spatiotemporal fragmentation.

Finally, as a comparison with our previous results obtained by employing the GONG and MDI data, we have plotted in Fig. 3 the radius of the top of the fragmentation region. This qualitative similarity of the fragmentation in all the cases is further evidence of robustness.

\section{Discussion}

We have shown that spatiotemporal fragmentation persists even when only the lowest order terms in the Legendre expansion of the inversion for the solar rotation law are used. This is important, as these terms appear to be approximately time independent (over the scale of a solar cycle), and also dominate the expansion. Indeed, these terms really represent the rotation law that should

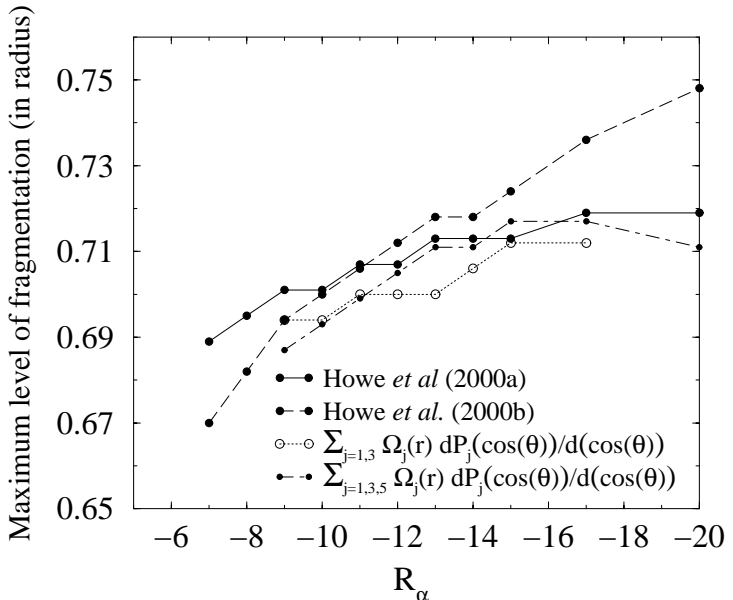

Fig. 3. The height of the top of the fragmentation region for different zero order rotation profiles $\Omega_{0}$ and different dynamo parameters $R_{\alpha}$.

be input to the dynamo code - in principle solution of Eq. (3) should then determine the variations of $\Omega$ over a cycle. Thus, these results allay worries that the inconsistency of averaging the observed rotation law over only a part of a solar cycle might be giving misleading results.

The results presented here, together with those of CTM1 and CTM2, show spatiotemporal fragmentation to be a very persistent feature of our simulations. This finding suggests that nonlinear dynamo modelling has a real potential predictive power, which could lead to a better understanding of the solar dynamo when longer data sets as well as improved analyses of them become available. This in turn, in conjunction with the expected improvements in the helioseismological data, could lead to an improved determination of the properties of the solar interior, including the nature of the tachocline.

\section{References}

Antia, H. M., \& Basu, S. 2000, ApJ, 541, 442

Covas, E., Tavakol, R., \& Moss, D. 2000a, A\&A, 363, L13

Covas, E., Tavakol, R., Moss, D., \& Tworkowski, A. 2000b, A\&A, 360, L21

Covas, E., Tavakol, R., \& Moss, D. 2001, A\&A, 371, 718

Howard, R., \& LaBonte, B. J. 1980, ApJL, 239, 33

Howe, R., et al. 2000a, ApJL, 533, 163

Howe, R., et al. 2000b, Science, 287, 2456

Kitchatinov, L. L., Mazur, M. V., \& Jardine, M. 2000, A\&A, 359,531

Kosovichev, A. G., \& Schou, J. 1997, ApJ, 482, 207

Moss, D., \& Brooke, J. 2000, MNRAS, 315, 521

Rüdiger, R., \& Brandenburg, A. 1995, A\&A, 296, 557

Schou, J., Antia, H. M., Basu, S., et al. 1998, ApJ, 505, 390

Snodgrass, H. B., Howard, R. F., \& Webster, L. 1985, Sol. Phys., 95, 221

Toomre, J., Christensen-Dalsgaard, J., Howe, R., et al. 2000, Sol. Phys., 192, 437

Vorontsov, S. V., Christensen-Dalsgaard, J., Schou, J., Strakhov, V. N., \& Thompson, M. J. 2001, in Helio- and Asteroseismology at the Dawn of the Millenium, ESA SP464,559 